\title{
Design of Multifunctional Hierarchical Space Structures
}

\author{
Christine Gregg \\ Dept. of Mechanical \\ Engineering \\ UC Berkeley \\ Berkeley, CA \\ cgregg@berkeley.edu
}

\author{
Benjamin Jenett \\ Center for Bits and \\ Atoms \\ MIT \\ Cambridge, MA \\ bej@mit.edu
}

\author{
Daniel Cellucci \\ Department of Mechanical \\ and Aerospace Engineering \\ Cornell University \\ Ithaca, NY \\ dwc238@ cornell.edu
}

\author{
Kenneth Cheung \\ NASA Ames Research Center \\ Moffett Field, CA \\ kenny@nasa.gov
}

\begin{abstract}
We describe a system for the design of space structures with tunable structural properties based on the discrete assembly of modular lattice elements. These lattice elements can be constructed into larger beam-like elements, which can then be assembled into large scale truss structures. These discrete lattice elements are reversibly assembled with mechanical fasteners, which allows them to be arbitrarily reconfigured into various application-specific designs. In order to assess the validity of this approach, we design two space structures with similar geometry but widely different structural requirements: an aerobrake, driven by strength requirements, and a precision segmented reflector, driven by stiffness and accuracy requirements. We will show agreement between simplified numerical models based on hierarchical assembly and analytical solutions. We will also present an assessment of the error budget resulting from the assembly of discrete structures. Lastly, we will address launch vehicle packing efficiency issues for transporting these structures to lower earth orbit.
\end{abstract}

\section{TABLE OF CONTENTS}

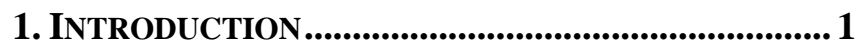

2. BACKGROUND............................................................ 2

3. METHOD............................................................... 4

4. RESULTS................................................................ 6

5. EVALUATION......................................................... 8

6. CONCLUSION................................................................99

ACKNOWLEDGEMENTS ..........................................99

REFERENCES............................................................ 9

BIOGRAPHY ...................................................................... 10

\section{INTRODUCTION}

Space Trusses

The necessity for large space structures has existed since the beginning of space exploration, with missions calling for energy collecting surfaces, habitats, and apertures for imaging or communication. Limited by mass/volume restrictions of the launch shroud, methods for achieving such structures can traditionally be divided into two categories: deployables and on-orbit construction.

Deployables tightly pack a structure to fit in a launch shroud, later unfurling into a larger configuration once in orbit. The unfurling mechanisms include articulating booms, umbrellalike antennas, unfolding star shades, and coilable masts. Complex deployment schemes for higher packing efficiency add more mass and volume for unfurling actuation, and lead to increased risk of error and mechanism failure [1]. This explains why most space systems experience highest failure rate during deployment [2]. Decades of refinement have reduced error rates, but anomalies are not uncommon, and are just as costly [3], [4], [5].

The second traditional approach is on-orbit construction, where parts are packed into a launch shroud and assembled in space by either robots or astronauts. While structures have been designed, actual implementation has been limited by construction methods available in orbit. Experiments have combined EVA (Extravehicular Activity) with EVR (Extravehicular Robotics) to create truss structures [6], translating platforms and articulated arms to locate "human end effectors"[7], and robotic arms with linear motion platforms, to make work envelopes [8]. On the ISS, telerobotic (human controlled) robotic arms have been trialed. However, the limitations in EVA (risk, time and efficiency challenges) and EVR (robotic complexity and locomotion challenges) continue to hinder extensive largescale on-orbit construction.

\section{Digital Materials}

A recent approach to the construction of large space structures is the use of modular, reconfigurable lattice elements known as digital materials. Reversibly-assembled digital cellular materials consist of a three-dimensional framework that has been decomposed into identical building blocks. These building blocks are assembled via a reversible mechanical connection to form continuous materials/systems with many desirable traits, including repairability, reconfigurability, and high-performing mechanical properties. These have been demonstrated in aerospace applications such as ultrastiff, ultralight materials [9], morphing aerospace designs [10] and reconfigurable, meterscale structures [11].

Digital lattice structures are distinguished from generalized on-orbit construction by their degree of modularity and periodicity, making them ideally suited for simplified robotic construction [12]. Rather than requiring complex robots with multiple degrees of freedom (DOF) required to perform several tasks, the approach taken for digital materials is to divide tasks between robots, and design these task-specific robots relative to their function and the structured environment in which they operate. This material-robot system can be coupled and optimized for construction, which is in contrast to the de-coupled examples which use standard robotic systems (ie: gantry and/or multi-DOF armature manipulator) and standard strut-and-node truss construction systems (intended for human assembly). 
It has been noted that construction of large space structures can benefit from commonality of structural elements, both for interoperability and replacement/repair [13]. This can also be thought of as versatility, where one element can perform multiple functions. However, when specific mission architectures are selected, it is apparent that structural requirements vary sufficiently to result in negative impacts of a one-size-fits-all approach. Lightly loaded elements may have unnecessary structural mass, and heavily loaded elements may be undersized.

In this paper, we will describe two main benefits of using digital materials to construct large space structures Figure 1. The first is the versatility of tunable mechanical properties through hierarchical construction. The second is an improvement over the state of the art in mass-based structural efficiency.
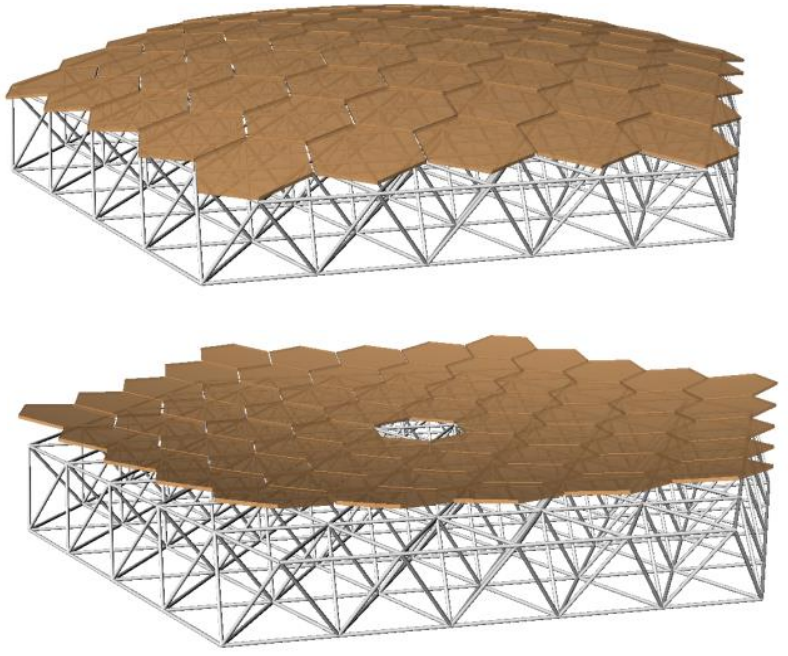

Figure 1: Large space structures. (Top) Aerobrake, (Bottom) Precision Segmented Reflector.

\section{BACKGROUND}

In this section, we present previous work and background on both case studies. Specifically, we wish to look at both examples in the context of the aforementioned methods for achieving large scale space structures- deployment and onorbit construction. Additionally, we will show the differences in the structural requirements for the two case studies. Last, we will describe two existing methods for digital material voxel construction.

\section{Aerobrake Structures}

An aerobrake is a large dish structure with thermal protection system (TPS) panels that enable a spacecraft to use a planet's atmosphere to decelerate for landing. This causes high thermal and pressure loads, but can ultimately reduce the amount of fuel required for propulsion during landing, and thus reduce the initial launch mass.

Typical aerobrake designs call for a rigid truss structure clad with TPS panels. However, due to the required scale of these aerobrakes for transportation of heavy cargo and/or spacecraft, numerous methods have been proposed to build aerobrakes larger than a launch vehicles payload volume. Inflatable aerobrake conical geometry can be decomposed into a number of structural elements, such as stacked tori, or spars with rims, which are rigidized by internal pressure [14].

Folding mechanisms that enable a large structure to pack tightly and then deploy have been proposed for aerobrakes [15]. Rather than pressurized rigidity, structural members compose a truss which simplifies analysis and design. The challenge, however, lies in the mechanism for actual deployment, as large structures require numerous systems to fit within a launch shroud. The last approach for large aerobrake structures is assembly of individual truss elements [16]. While this approach removes the complexity of deployment, it requires complex robotic systems to enable construction. We build upon this approach by introducing digital materials for discrete construction, enabled and simplified by relative robotic systems.

Realized experiments of aerobrakes include the Inflatable Reentry Vehicle Experiment [17], which successfully demonstrated deployment and re-entry. Also successfully tested is an umbrella-like system on the IRENE space capsule [18]. There are no examples of discretely assembled aerobrake structures being tested.

\section{Precision Segmented Reflector Structures}

The design requirements of precision reflectors are typically characterized by light structural loading and extreme structural accuracy (driven by electromagnetic requirements of wavelength being reflected [19]). Traditional design considerations of assembled precision reflectors are well reported by Mikulas in [20] and [21], investigating design drivers of fundamental frequency, packing efficiency, assembly time, and weight. Properties relating natural frequency, as a function of structural mass and geometry, to surface accuracy were explored in [22] [23] [24]. This approach is used later in this paper.

There are currently no precision segmented reflectors in operation. The James Webb Space Telescope will deploy a $25 \mathrm{~m} 2$ aperture and a tennis court-sized sunshield and must fit within a $4.5 \mathrm{~m}$ diameter launch shroud [25]. We do find numerous examples of deployable dishes with lower precision requirements [26] [27], but there have been no such dishes employing on-orbit construction .

Since reflectors are too large to launch fully assembled, the error from assembly and manufacturing tolerances can be a primary source of error. Because of this, tight manufacturing tolerances are typically necessary. Bush [28] presented the design and fabrication of an erectable truss reflector that achieved a surface accuracy of 0.003 in. (rms). To achieve this, it was necessary that each truss strut was carefully manufactured and measured to a tolerance of $0.0002 \mathrm{in}$. The effect of manufacturing tolerances on truss accuracy has also been studied analytically and computationally. Greene 
simulated the effects of random member length of the surface accuracy and defocus of a tetrahedral truss antenna reflector [19], finding that increasing the number of rings in the truss (number of members) significantly decreased surface error and defocus. It was also found that increasing the number of hexagonal rings increased agreement with continuum estimates presented in [29], which related the rms surface error for a given part error distribution with the vibrational modes of the structure.

In the present study, we seek to extend previous work on manufacturing tolerance effect on accuracy by investigating such affects for trusses constructed from digital materials. We argue that these hierarchical structures have multiple mechanisms for increasing the precision of the resulting structure above that of component parts. This is accomplished primarily through two different ideas: statistical averaging and elastic averaging. Statistical averaging refers to the tendency of errors in a population to be 'averaged out' when summed. Elastic averaging refers to the ability of overconstrained systems to deform elastically and average errors. We investigate the effects of these types of averaging through a scaling argument and finite element analysis.

To highlight the versatility of our construction system, we can juxtapose the structural systems and requirement for the aerobrake and precision segmented reflector, the former being driven by strength requirements and the latter being driven by stiffness and precision requirements. It is noted in [21] that loading on PSR struts while in operation is quite low, and that the main structural considerations are residual stress from strut length imperfections and CTE variations. For instance, [21] describes Euler buckling capacity on the order of $1000 \mathrm{lb}(4.45 \mathrm{kN})$, while [16] shows for an aerobrake that even the most lightly loaded struts have axial loads up to $10,000 \mathrm{lb}(44.5 \mathrm{kN})$.

Such diversity of structural requirements demonstrates why a single traditional construction kits of struts cannot be used to build both structures, despite their similar tetrahedral plate design. Struts sufficient for the aerobrake would be greatly over-engineered and wasteful for use in the precision reflector. We will show that due to the reconfigurability, hierarchical construction of digital materials, we can use the same basic set of building blocks to achieve a wide range of structural properties.

\section{Digital Material Voxel Construction}

Our base structural system is a Cuboct lattice, made of vertex connected octahedron. These are referred to as voxels, or volumetric pixels, because they can be used to fill 3D space. There are a number of ways to construct the voxels- we will investigate and compare two: injection molding and discrete assembly. Injection molding is a highly repeatable process with low cost and high throughput. However, limitations exist as far as mold complexity for 3D shapes. Injection molding allows for high stiffness materials such as glass fiber reinforced plastics. The voxels are joined with nuts and bolts which are sized based on the expected load requirements. This allows them to be reversibly assembled, while also assuring sufficient load transfer and rigidity at the joints.
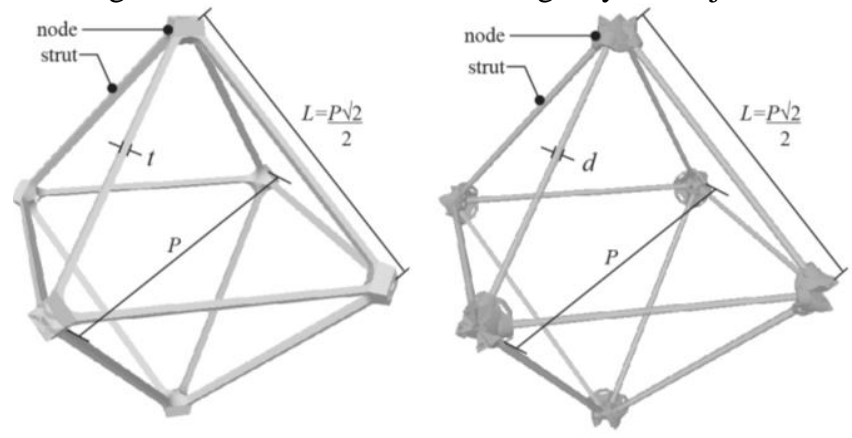

Figure 2: Comparison of voxel production methods. (L) Injection molded, (R) Discretely Assembled.

An alternative approach is discrete assembly [11]. This approach utilizes individual struts and nodes to construct the voxel. In this case, the struts are unidirectional pultruded carbon fiber with a Young's modulus of $130 \mathrm{GPa}$. This, in addition to the ability to use hollow tubes, provides opportunities for higher stiffness to weight ratios than injection molding. However, nodes for this version are larger due to alignment features for assembly, resulting in more parasitic mass, which reduces specific stiffness. As we will show, this tradeoff is fairly balanced at small scales $\left(L \approx 10^{2}\right.$ $\mathrm{mm})$, but at larger scales $\left(L \approx 10^{3}-10^{4} \mathrm{~mm}\right)$, discrete assembly offers higher overall specific stiffness values. The last consideration between these two options is packing efficiency, which will be addressed in later sections.
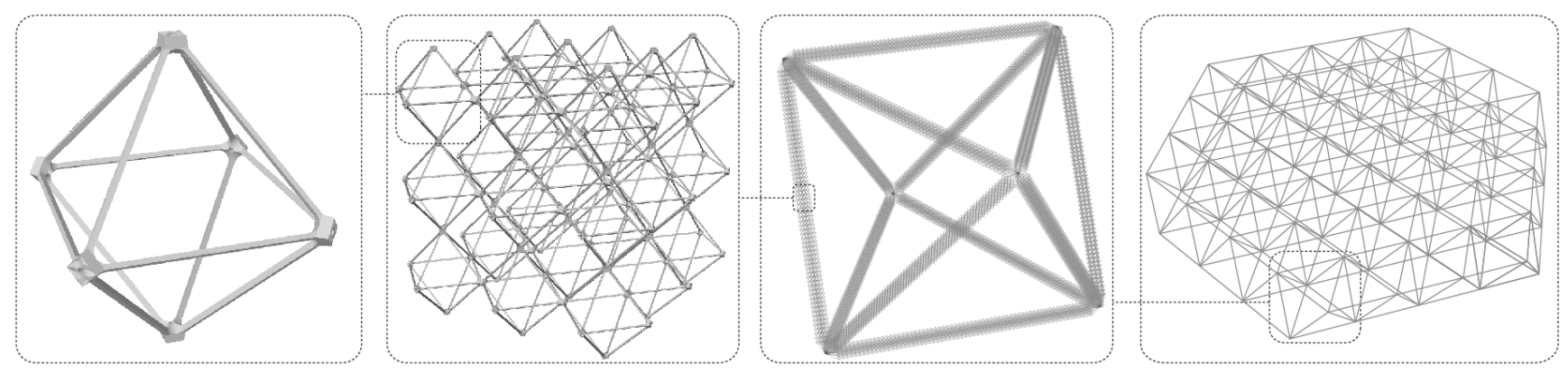

Figure 3: Overview of hierarchical discrete lattice system. ( $L$ to $R$ ) Individual voxel, 3x3x3 voxel cube, tetrahedra using $3 \times 3$ voxel struts, tetrahedral space structure build from $3 \times 3$ strut elements 


\section{METHOD}

We describe now the steps for design of multifunctional hierarchical space structures (Figure 2). We first look at the effects of discrete assembly on the precision of larger structures, as defined by the error within individual voxels and the cumulative effect of elastic averaging of these errors. We then we describe leveraging the modularity of the structure to simplify analysis methods through hierarchical representation. Lastly, we describe the tetrahedral dish geometry to be analyzed for both the aerobrake and the precision segmented reflector.

\section{Statistical Averaging of Hierarchical Structures}

Here we present a simple one-dimensional scaling argument for understanding how beam error should scale with the error of component parts. In this exercise, assume that we have a population of parts with lengths that are normally distributed with a known mean $\mu$ and standard deviation $\sigma$. To simplify this problem, we assume that we are dealing with a $1 \times 1$ voxel beam cross-section, avoiding effects of elastic averaging that will be addressed in the following section. We are interested in the error that can be expected in the length of a beam made from a given number of parts taken from this population.

If the length of single part is $\mathrm{x}$, the length of a beam $\mathrm{L}$ with $\mathrm{n}$ parts is given by

$$
L=\sum_{i=1}^{n} x_{i}
$$

Since the mean of a sample of $\mathrm{n}$ parts is given by

$$
\bar{x}_{n}=\frac{\sum_{i=1}^{n} x_{i}}{n}
$$

the length of the bar can also be written as

$$
L=n \bar{x}_{n}
$$

Therefore, to characterize the precision of the length of a beam constructed from $n$ parts, we are concerned about the behavior of the sample mean as a function of $n$. Because of this, we calculate the Standard Error of the Mean, which is the standard deviation of the sample-mean's estimate of the population mean. This is given by

$$
S E_{\bar{x}}=\frac{\sigma}{\sqrt{n}}
$$

where $\sigma$ is the population standard deviation. It is well known in statistics that the means of samples of a population form a normal distribution about the population mean. The standard error of the mean is the standard deviation of this distribution. Figure 4 shows this behavior for three prototypical population standard deviations.

Since we are dealing with a normally distributed population, we can define $99 \%$ confidence intervals for the mean of

$$
\text { mean }=\bar{x} \pm 2.58 S E_{\bar{x}}
$$

Similarly, we can write the length of the beam $\mathrm{L}$ as

$$
L=n\left(\bar{x} \pm 2.58 S E_{\bar{x}}\right)
$$

If we want to understand the expected percentage error of the length for given number of parts with $99 \%$ confidence

$$
\text { Percent Error }=\frac{2.58 S E_{\bar{x}}}{\bar{x}}
$$

where it should be remembered that $S E_{\bar{x}}$ is a function of $\mathrm{n}$.

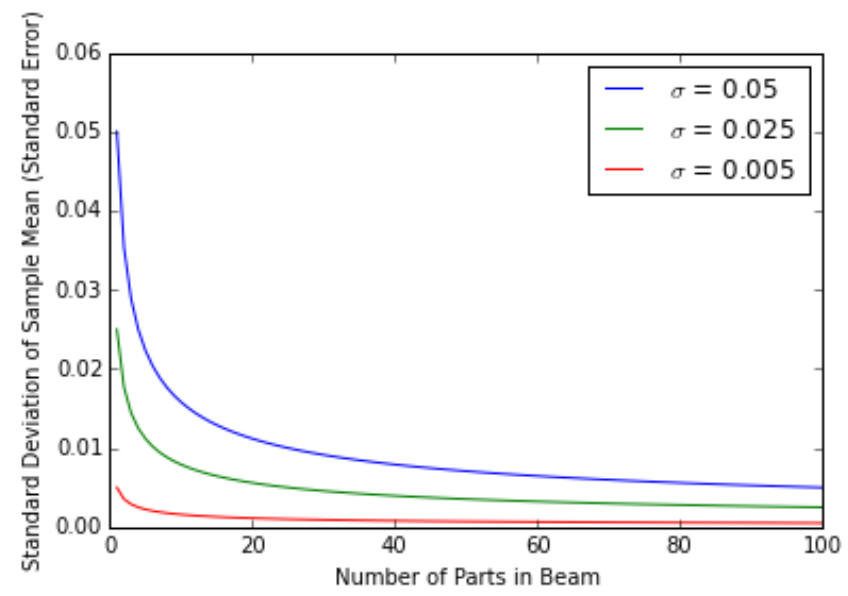

Figure 4: Standard Deviation of Beam Length for Multiple Component Standard Deviations. Plot shows the dependence of the beam standard deviation on the number of component parts (length) and original part length standard deviation

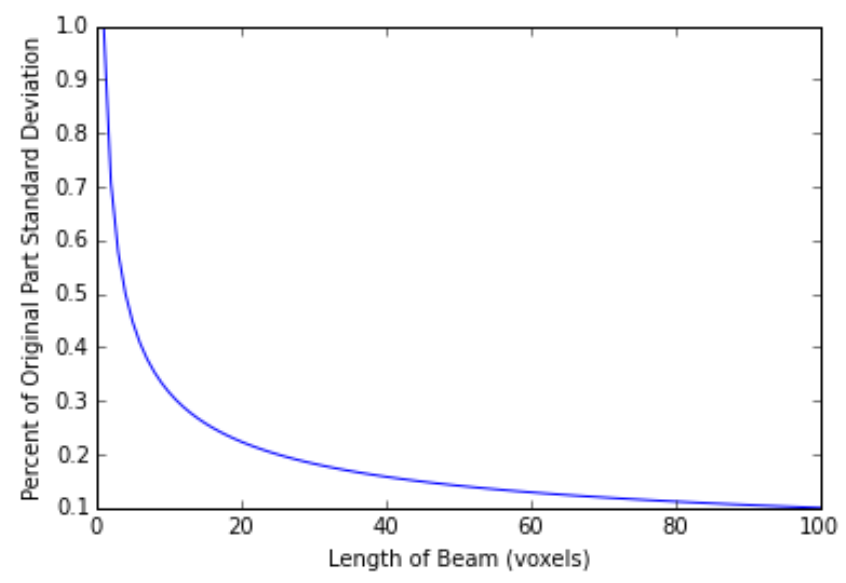

Figure 5: Percent Reduction of Beam Standard Deviation from Component Standard Deviation. Composite beam standard deviation as a percentage of component part standard deviation for given beam length (number of component parts). Notice that at 30 component parts, the standard deviation of the assembled beam is predicted to be 20 percent of the part population standard deviation. 
From this argument, we can expect increased precision with an increase in parts. In the proposed reflector design, truss beams are on the order of 50 parts long. From Figure 4, it can be seen that the standard deviation of the assembled 50 parts beam can be expected to be only $20 \%$ of the standard deviation of the constituent parts ( $80 \%$ increase in precision).

\section{Elastic Averaging of Hierarchical Structures}

To investigate the effects of elastic averaging on assembled beam precision, FEA simulations of randomized truss member length errors were conducted using ABAQUS 6.14. It was proposed in [30] that randomized element length errors can be simulated by assigning each exact length element in the model a random coefficient of thermal expansion and subjecting the structure to a temperature increase. Such a simulation was conducted for $1 \times 1 \times 10,2 \times 2 \times 10$, and $3 \times 3 \times 10$ cuboct beams.

We wish to simulate the effects of random manufacturing error on truss beam length precision, assigning a random length error $e$ to each truss element. If the nominal beam length is $L^{*}$, then

$$
\text { Error }=e=L-L^{*}
$$

This can be conceptually transformed into an equivalent random initial strain in each truss element [30].

$$
\epsilon_{i}=\frac{L-L^{*}}{L^{*}}=\frac{e}{L^{*}}
$$

If the error is normally distributed about an average with a given standard deviation $\sigma_{e}$, then for a $99 \%$ confidence interval, the initial strain can be written as

$$
\epsilon_{i}=\frac{\bar{e} \pm 2.58 \sigma_{e}}{L^{*}}=\bar{\varepsilon}_{i} \pm 2.58 \sigma_{\varepsilon_{i}}
$$

In our case, the average of the error is zero (error equally likely to be negative and positive). Thus the initial strain should also be centered about zero.

One method of achieving strain is orthotropic thermal expansion along the beam length (coefficient of thermal expansion is zero in plane of beam cross-section). We know that the strain of thermal expansion is dependent upon the coefficient of thermal expansion $\alpha$ and the change in temperature $\Delta T$ :

$$
\epsilon_{T}=\alpha \Delta T
$$

If the coefficient of thermal expansion is distributed about an average with a given standard deviation $\sigma_{\alpha}$, it can be shown that

$$
\epsilon_{T}=\left(\bar{\alpha} \pm 2.58 \sigma_{\alpha}\right) \Delta T=\bar{\varepsilon}_{T} \pm 2.58 \sigma_{\varepsilon_{T}}
$$

By equating the thermal strain and the desired initial strain, it can be shown that

$$
\sigma_{\varepsilon_{i}}=\left(\sigma_{\alpha}\right) \Delta T=\frac{\sigma_{e}}{L^{*}}
$$

In this study, a lattice pitch of 3 inches was used, and a thermal expansion coefficient standard deviation of $0.1 /$ strut length $=0.47$ was implemented with a unity change of temperature. From the preceding expression, this corresponds to an error standard deviation of 1.414 inches for a beam length of 10 voxels (30in). Ten trials were conducted for each cross-sectional area. Deformed beam length was defined as the average lengthwise dimension of nodes at the end of the beam.

\section{Hierarchical Analysis Descriptions}

It has been shown that discretely assembled structures can employ a method of simplification called "Physical Finite Element Analysis" (PFEA) [31]. Because we have physical access to the building blocks which constitute the larger structure, we can empirically test these to calibrate model parameters rather than rely on bulk material models.

Prior work has shown that modularity in construction permits a description of the continuum as a series of beams and nodes, which can be used to create a voxel-based, tuned mass-spring lattice model to simulate the dynamics of highly deformable heterogeneous materials [32]. In homogeneous, periodic volumes, the behavior of networks of Euler-Bernoulli beams can be used to efficiently model bulk material behavior [33]

We apply a similar method here, but rather than physically tuning our model, we apply simple Euler Bernouli beam model FEA to extract behavior of hierarchical, multi-voxel macro struts. The properties of these assemblies, including structural mass, parasitic mass, bending stiffness, and specific stiffness, are then used to model larger structures.

Rather than create computationally expensive models with thousands of parts, we can hierarchically verify constructs which get abstracted at higher levels. This approach offers benefits when large scale structures are too difficult to experimentally verify, which is common for very long composite elements such as the struts used in our reviewed literature. Our approach decomposes large structures into smaller, verifiable elements which can then be used as data in analytical and numerical modeling.

\section{Tetrahedral Dish Design}

The same basic tetrahedral dish design is used for both the aerobrake and the precision segmented reflector, though different hierarchical cross sections are optimized based on each application's specific structural requirements. The analysis used in Dorsey [16] is recreated here, incorporating the previously described approach of hierarchical modeling using digital materials. We make similar assumptions:

-Truss diameter: $36.576 \mathrm{~m}(120 \mathrm{ft})$.

-Truss depth: $3.54 \mathrm{~m}(11.6 \mathrm{ft})$

-Strut length: $4.33 \mathrm{~m}(14.2 \mathrm{ft})$ 


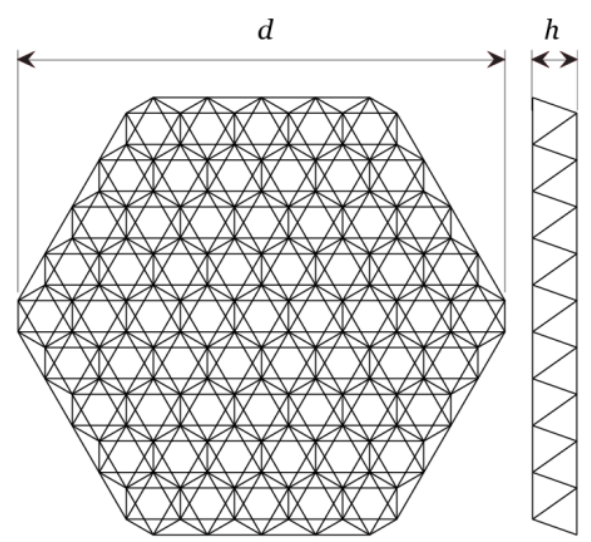

Top View

Side View

Figure 6: Schematic view of main truss dimensions

We do not address panel design here, although this is a topic for further optimization. The analysis software used for this case study was Oasys GSA, which is capable of analyzing Euler-Bernoulli beam networks.

\section{RESULTS}

\section{Aerobrake}

As previously stated, design of the aerobrake was driven by structural loading of the truss struts. Additional parameters for the analysis of this application are listed below:

-Attached spacecraft mass: 204,000 kg (450,000 lb).

-Deceleration rate: 6 g's

-Resulting uniform panel pressure: $13.79 \mathrm{kPa}$ (2.1 psi)

-Safety factor: 1.4.

-\# of attachment points to spacecraft: 6 .

The results of our linear-elastic FEA simulation, performed in Oasys GSA software, are show in Figure 7.

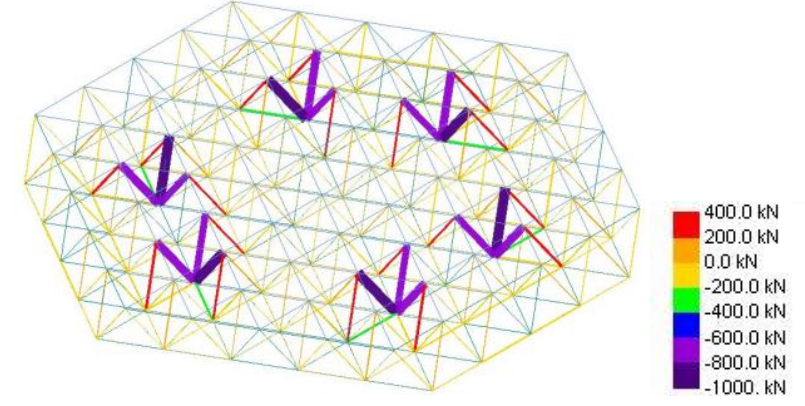

Figure 7: Simulation results for aerobrake. Axial forces shown with $13.79 \mathrm{kPa}$ pressure loading, 204,000 kg payload, and 6 point of contact to spacecraft.

TABLE I.

AXIAL FORCE MEMBER GROUPING

\begin{tabular}{cc}
\hline Axial Force & Number of Members \\
\hline $250-1000 \mathrm{kN}$ & 18 \\
$125-250 \mathrm{kN}$ & 94 \\
$0-125 \mathrm{kN}$ & 488 \\
\hline
\end{tabular}

We can use the axial forces to guide the design the macro- struts in various sizes. The minimum cross section for a column is $2 \times 2$ voxels. We will focus on a $5 \times 5$ cross section to design the highest loaded struts, which receive loads up to $1000 \mathrm{kN}$. Based on the geometry of the octahedron, it can be determined that for an axial force $F$ applied to the node along $x, y$, or $z$ axis, the resulting axial forces in the struts will be $0.35 \cdot F$. The $5 \times 5$ distributes its load between 25 voxels, resulting in a voxel load of $40 \mathrm{kN}$ and a strut load of $14 \mathrm{kN}$. For the initial design of the strut, we can consider Euler buckling, which will provide us with a required area moment of inertia, $I$, and thus we can arrive at a cross sectional area and tube shape. We can rearrange $F=\frac{\pi^{2} E I}{(K L)^{2}}$ to solve for $I:=$ $\frac{F *(K L)^{2}}{\pi^{2} E}$. Using $F=14 \mathrm{kN}, E=130 \mathrm{GPa}, L=45 \mathrm{~mm}$, and $K$ $=0.7$ (found empirically in [11]), we find that $I=10 \mathrm{~mm}^{4}$. Looking at practical cross sections, we find that a tube with outer diameter $D=4 \mathrm{~mm}$ and wall thickness $t=0.65 \mathrm{~mm}$ will be sufficient.

It is also important to analyze the struts for failure in tension. We can find the axial stress by dividing $14 \mathrm{kN}$ by the cross sectional area, $6.8 \mathrm{~mm}^{2}, \sigma_{A}=14 \mathrm{kN} / 6.8 \mathrm{~mm}^{2}=2 \mathrm{GPa}$. We find that unidirectional carbon fiber with an epoxy matrix has a tensile strength of up to $2.17 \mathrm{GPa}$. This is sufficient for our design. Looking at the other two axial force groups, we find that a $3 \times 3$ will satisfy the $125-250 \mathrm{kN}$ group, as the maximum axial force is $11.6 \mathrm{kN}$, and a $2 \times 2$ will satisfy the 0 $125 \mathrm{kN}$ group, as the maximum axial force is $10.94 \mathrm{kN}$.

We can then design the nodes and hardware. In order to sufficiently capture struts of this diameter, each node has a diameter of $12.5 \mathrm{~mm}$, resulting in a total node mass of $7.5 \mathrm{~g}$. In order to connect voxels, a bolt must be able to withstand up to $40 \mathrm{kN}$ (9000 lbf) in tension. This can be accomplished with a M7-12.9 bolt. The cumulative hardware mass for each voxel, then, is $17 \mathrm{~g}$. Each strut weighs $0.46 \mathrm{~g}$, for a total of $5.5 \mathrm{~g}$, and the resulting voxel mass is $30 \mathrm{~g}$. The total number of voxels for the aerobrake is 166,896 .
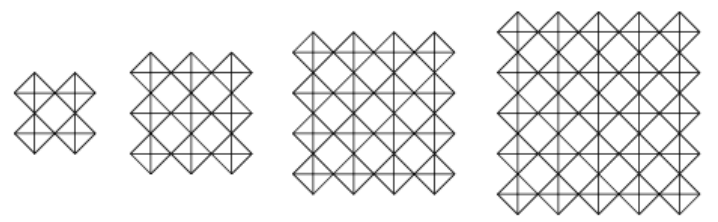

Figure 8: Hierarchical voxel macro-strut cross sections. (L to $R$ ) $2 \times 2,3 \times 3,4 \times 4,5 \times 5$.

\section{PSR: Structural and Modal Analysis}

Following the aerobrake design, we select $2 \times 2$ beams for the PSR, due to the fact that the truss will be very lightly loaded and will be driven by stiffness and precision requirements. We will first address the stiffness requirements. We can observe from [24] that surface precision of a dish is driven primarily by the inverse square of the natural frequency:

$$
\delta \propto 1 / f_{0}^{2}
$$


Where $f_{0}$ is the fundamental frequency, and $\delta$ is the rms surface deformation, which we seek to minimize. It has been shown in [22] that aiming for a $f_{0}$ around $10 \mathrm{~Hz}$ is desirable, to accommodate most expected disturbances passively, so this is what our objective will be. We will now apply analytical approaches developed in [24] to find the modal response of a digital material tetrahedral plate for the application of a precision segmented reflector (PSR).

$$
\left(f_{0}\right)_{\text {segmented }}=(0.852 / d)(h / d) \sqrt{\eta(E / \rho)_{\text {truss }}}
$$

Where, $d$ is truss diameter, $h$ is truss height, $\eta$ is the ratio of truss mass to total mass, $E / \rho$ is the specific stiffness of the truss. We find that $f_{0}=7.369 \mathrm{~Hz}$. We then employ our hierarchical modeling approach to create a simplified FEA model for simulation of modal analysis.

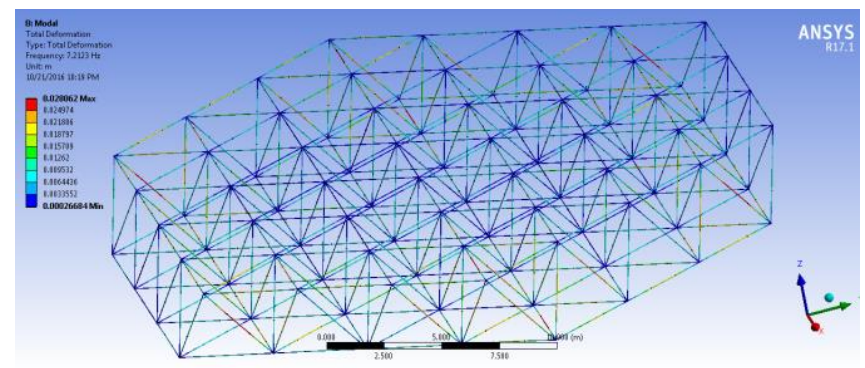

Figure 9: Simulation results of free-free modal analysis. Software used is ANSYS.

TABLE II. FEA Modal RESULtS FOR FREE-FrEe ANALYSIS

\begin{tabular}{cc}
\hline Mode & Frequency $(\mathbf{H z})$ \\
\hline 1 & 0 \\
2 & 0 \\
3 & 0 \\
4 & 0 \\
5 & 0 \\
6 & $7.3 \mathrm{e}-6$ \\
7 & 7.2123 \\
\hline
\end{tabular}

For a free-free analysis, we expect the first 6 modes for be rigid body modes (translation $x, y, z$, rotation $x, y, z$ ), so the $7^{\text {th }}$ mode is the lowest natural frequency, $7.212 \mathrm{~Hz}$. When we compare this to the analytical solution, $7.369 \mathrm{~Hz}$, we find that they are within $2 \%$ of each other. This near the desired natural frequency of $10 \mathrm{~Hz}$. Based on using $2 \times 2$ voxel beams, we find that the PSR requires 133,377 voxels.

\section{PSR: Elastic Averaging and Precision}

Figure 10 shows the results from FEA simulations of random strut error on the length of truss beams of different cross sections of $n \times n$ voxels $(1 \times 1,2 \times 2,3 \times 3)$. It can be seen that the average absolute beam error decreases with increasing $n$, as does the standard deviation of the error (using a 3x3 instead of a $1 \times 1$ decreased standard deviation by 80 percent) (see Table III). This demonstrates the potential of tuning truss beam precision while maintaining a constant truss beam design length. Additionally, increasing beam cross section will add more structural mass, thereby further lowering natural frequency modes [22]

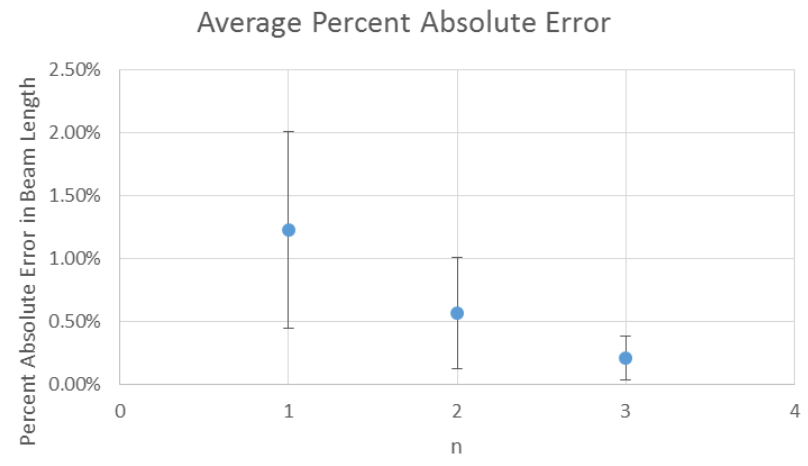

Figure 10: Percent Beam Length Error for Randomized Strut Error. Percent absolute error in beam length for truss beam with an $n \times n$ voxel cross section. Error bars represent a single standard deviation.

TABLE III. AVERAGE ERRORS FOR GIVEN CROSS-SECTION

\begin{tabular}{ccccc}
\hline $\mathbf{n}$ & $\begin{array}{c}\text { Average } \\
\text { Abs. Error }\end{array}$ & $\begin{array}{c}\text { Average Abs. } \\
\text { Error STD }\end{array}$ & $\begin{array}{c}\text { Average \% } \\
\text { Error }\end{array}$ & $\begin{array}{c}\text { Average \% } \\
\text { Error STD }\end{array}$ \\
\hline 1 & 0.3678 & 0.2338 & 0.0123 & 0.0078 \\
2 & 0.1694 & 0.1331 & 0.0056 & 0.0044 \\
3 & 0.0624 & 0.0516 & 0.0021 & 0.0017 \\
\hline
\end{tabular}

\section{Launch Vehicle Packing Efficiency}

Since we have designed large space structures with thousands of voxels, we need to assess the best way to transport these into space. We now compare the packing efficiency between the two manufacturing methods. We can fill a volume with tightly packed injection molded voxels, as shown in Figure 11. We can define this volume as a function of strut length $L$. We see that 64 voxels pack within a volume of $65.8 \cdot L^{3}$, resulting in a per voxel packing volume of roughly $1 \cdot L^{3}$.

Discrete assembly of voxels allows struts and nodes to be packed together more efficiently, as shown in Figure 11. For a given voxel with strut length $L$, we find that 12 struts and 6 nodes can pack into a volume of $0.0675 \cdot L^{3}$. This is 15 times more efficient than the injection molded approach. However, this approach will require a robot/machine that can assemble voxels prior to the robotic assembly of macro-struts, to be addressed in future work
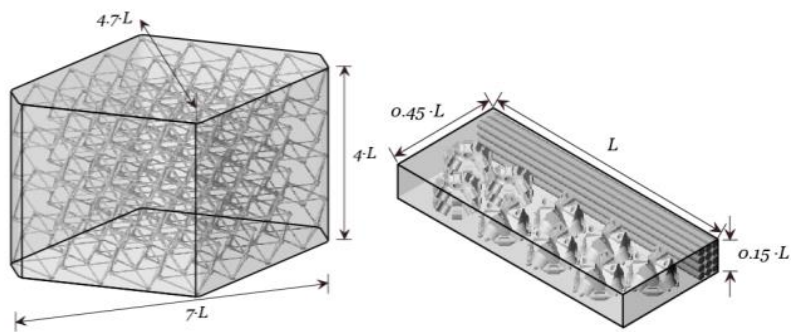

Figure 11: Comparison of voxel packing volumes. (L) Injection molded (IM), 64 voxels shown. (R) Discrete assembly (DA), 1 voxel shown. 
We assess this comparison in more detail by selecting points of comparison. First, we will look at the specific voxels we are currently producing through injection molding. We have produced injection molded voxels with a lattice pitch $P=$ $76.2 \mathrm{~mm}$ (3.0") and a strut length $L=\mathrm{P} \sqrt{2} / 2=53.88 \mathrm{~mm}$ (2.12"). The strut has a square cross section with a side length $\sim \mathrm{L} \sqrt{2} / 64=1.5 \mathrm{~mm}(0.056 ")$. The material is Zytel with $30 \%$ chopped glass fiber reinforcement, with a Young's modulus $E=10 \mathrm{GPa}$. We can design a voxel with the same relative stiffness from struts and nodes. The result is a carbon fiber tube with outside diameter $D$ of $1.25 \mathrm{~mm}$ and wall thickness $t$ of $0.15 \mathrm{~mm}$. The node mass is $4.5 \mathrm{~g}$, giving an overall voxel mass of $5 \mathrm{~g}$. This is twice the mass of the injection molded voxel, which is attributable to the parasitic mass at the node to enable assembly.

We can now take a sample launch vehicle (LV), in this case a Falcon 9. This LV has a payload capacity of $275 \mathrm{~m}^{3}$ and $13,000 \mathrm{~kg}$. We show the results in Table IV.

TABLE IV.

76MM LATTICE PITCH PACKING EFFICIENCY

\begin{tabular}{ccccc}
\hline Method & Mass $(\mathbf{k g})$ & Volume $\left(\mathbf{m}^{\mathbf{3}}\right)$ & Limit & Quantity \\
\hline Injection Molding & 6188 & 275 & Vol. & $2.38 \times 10^{6}$ \\
Discrete Assembly & 13,000 & 14 & Mass & $2.6 \times 10^{6}$ \\
\hline
\end{tabular}

Both methods are able to pack roughly the same amount, but one is mass limited and one is volume limited. This indicates that at such a small scale, the difference between the two methods is less significant. To compare with a larger voxel size, we will now design an injection molded part to match the voxel presented in [11], which has the following properties: lattice pitch $P=283 \mathrm{~mm}$, strut length $L=175 \mathrm{~mm}$, tube diameter $\mathrm{D}=5 \mathrm{~mm}$, wall thickness $t=1 \mathrm{~mm}$, Young's modulus $E=130 \mathrm{GPa}$, with an overall voxel mass of $115 \mathrm{~g}$. We find that the equivalent injection molded voxel will have a strut with a circular cross section with diameter $D=9 \mathrm{~mm}$, resulting in an overall voxel mass of $235 \mathrm{~g}$. We can then compare the packing efficiency, as shown in Table $\mathrm{V}$.

TABLE V. 283MM LATTICE PITCH PACKING EFFICIENCY

\begin{tabular}{ccccc}
\hline Method & Mass $(\mathbf{k g})$ & Volume $\left(\mathbf{m}^{\mathbf{3}}\right)$ & Limit & Quantity \\
\hline Injection Molding & 10,973 & 275 & Vol. & 46,694 \\
Discrete Assembly & 13,000 & 40 & Mass & 113,043 \\
\hline
\end{tabular}

Here we can see that discrete assembly results in more than twice as many voxels. This is attributable to the fact that as the voxel size increases, the benefit of hollow tubes over solid rods for struts becomes more significant, as well as the effects of voxel scale relative to launch shroud dimensions.

Additionally, we can view these packing results in combination with mass and volume estimations for hexagonal panels (either mirror or thermal protection system). The PSR panels weigh a total of 2,500 kg [22], with $4,000 \mathrm{~kg}$ of structures, for a total of $6,500 \mathrm{~kg}$. The AB panels weigh a total of $5,000 \mathrm{~kg}$ [16], with $5,000 \mathrm{~kg}$ of structures, for a total of $10,000 \mathrm{~kg}$. These results are shown in Figure 12.

\section{Digital Material Launch Packing Efficiency}

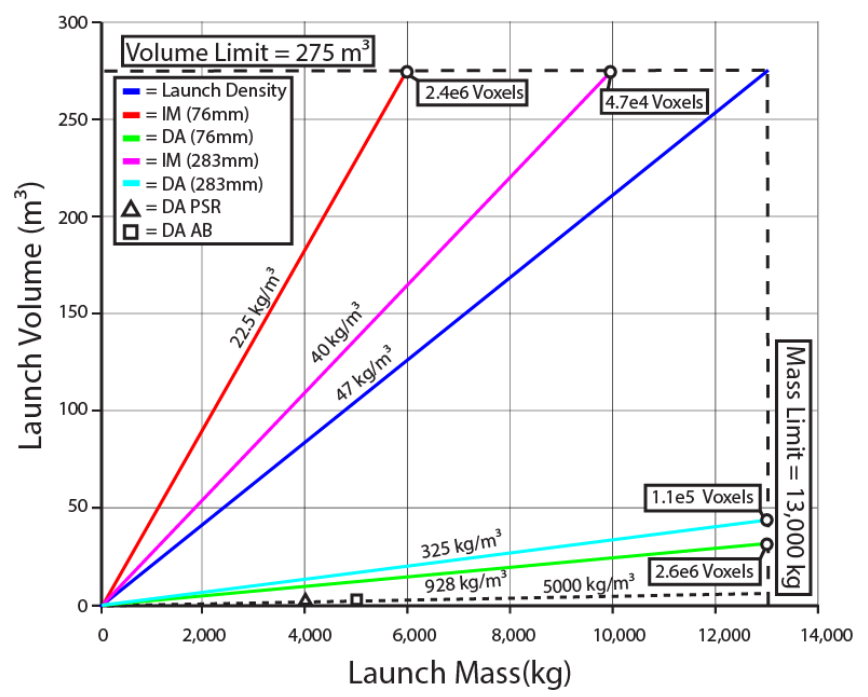

Figure 12: Comparison of packing efficiency of digital material structures. Shown are plots for $76 \mathrm{~mm}$ pitch lattice and $283 \mathrm{~mm}$ pitch lattice in both injection molded (IM) and discretely assembled (DA) versions, results from design of digital material aerobrake and precision segmented reflector (PSR). Assumed launch vehicle is Falcon 9 to $\mathrm{LEO}$ with $13,000 \mathrm{~kg} / 275 \mathrm{~m}^{3}$ capacity.

\section{Evaluation}

We can compare the results of our aerobrake and precision segmented reflector designs to others in literature. We assume that mirror panels and thermal protection system panels are similar, and thus can be ignored. We will compare truss mass for a given comparable performance.

The digital material aerobrake structure mass is approximately $5,000 \mathrm{~kg}(11,000 \mathrm{lb})$. Results from [16] for similar geometry and loading conditions range from 12,000 $\mathrm{lb}$ to $18,000 \mathrm{lb}$, depending on how joint mass is calculated.

Our precision segmented reflector has a total mass of $4000 \mathrm{~kg}$. A comparable truss as described in [22] would have a mass of approximately $10,000 \mathrm{~kg}$. It should be noted that the referenced design was for $25 \mathrm{~m}$, and the areal density was used to extrapolate the mass at $40 \mathrm{~m}$.

Combining the effects of statistical and elastic averaging in our truss design, assuming a $2 \times 2$ cross-section and $\sim 50$ part length, the beams constructed from our parts can be expected to be approximately an order of magnitude more precise than the constituent parts ( $80 \%$ precision increase from statistical averaging, $40 \%$ precision increase from elastic averaging in $2 \times 2$ ), greatly reducing manufacturing tolerance requirements. However, it is important to note that additional precision could be achieved by moving to a $3 \times 3$ cross section of the same parts (though at the expense of mass). Considering the difficulty high manufacturing tolerances add to assembly and manufacturing of these structures, as evidenced by [28], this method of reducing necessary tolerances renders DLMs a 
promising strategy for increasing manufacturing ease, speed, and feasibility.

Digital materials can be packed into different launch configurations, with varying volume and mass constraints. Based on the mission, this can inform the selection of voxel manufacturing methods. The packing efficiency ratio can be nearly $2 \%$ of the volume of the final structure, which is competitive with the current best practice [34].

\section{Conclusion}

In the preceding work, we presented a single structural system capable of achieving hierarchical designs of two different large space structures with vastly different performance criteria. By doing so, we demonstrated the ability of these digital lattice materials to tune structural parameters based on design needs. In the case of the aerobrake, it was shown that we can perform as well as a traditional truss structure while potentially saving mass by using hierarchical assembly to tune strength parameters. In the case of the precision reflector, it was shown that the precision of hierarchical truss beams (and therefore overall structural precision) could be controlled through increasing voxel cross-sectional area and number of voxels per beam length. Future work will include experimental validation of precision models, as well as optimization of voxels for space applications.

\section{ACKNOWLEDGEMENTS}

This work was supported by the NASA Space Research Technology Fellowship (NSTRF) grants NNX16AM65H NNX13AL38H, and NNX14AM40H, and NASA ARMD CAS Mission Adaptive Digital Composite Aerostructures Technologies (MADCAT).

\section{REFERENCES}

[1] J. J. Watson, T. J. Collins, and H. G. Bush, "A history of astronaut construction of large space structures at NASA Langley Research Center," in Aerospace Conference Proceedings, 2002. IEEE, 2002, vol. 7, pp. 7-3569-7-3587 vol.7.

[2] R. Freeland, G. Bilyeu, G. Veal, and M. Mikulas, "Inflatable deployable space structures technology summary," 49th International Astronautical Congress. 1998.

[3] G. Tsuyuki and R. Reeve, "Galileo High-Gain Antenna Deployment Anomaly Thermal Analysis Support," J. Thermophys. Heat Transf., vol. 9, 1995.

[4] D. S. Adams and M. Mobrem, "Analysis of the lenticular jointed MARSIS antenna deployment," in 47th AIAA/ASME/ASCE/AHS/ASC Structures, Structural Dynamics, and Materials Conference, 2006, no. May, pp. 1-13.

[5] M. Steiner, "Spartan 207/Inflatable Antenna Experiment - Preliminary Mission Report," NASA Goddard Sp. Flight Cent., 1997.

[6] I. Bekey, "Space Construction Results: The
EASE/ACCESS Flight Experiment," Acta Astronaut., vol. 17, 1988.

[7] H. Bush and M. Lake, "The Versatility of a Truss Mounted Mobile Transporter for In-Space Construction," NASA Tech. Memo. 101514, 1988.

[8] W. R. Doggett, "Robotic Assembly of Truss Structures for Space Systems and Future Research Plans," in IEEE Aerospace Conference Proceedings, 2002.

[9] N. Cheung, Kenneth C.; Gershenfeld, "Reversibly Assembled Cellular Composite Materials," Science (80-. )., vol. 341, no. September, pp. 1219-1221, 2013.

[10] B. Jenett, K. C. Cheung, and S. Calisch, "Digital Morphing Wing: Active Wing Shaping Concept Using Composite Lattice-based Cellular Structures," Soft Robot., vol. 3, no. 3, 2016.

[11] B. Jenett, D. Cellucci, C. Gregg, and K. C. Cheung, "Meso-scale digital materials: modular, reconfigurable, lattice-based structures," in Proceedings of the 2016 Manufacturing Science and Engineering Conference, 2016.

[12] B. Jenett and K. C. Cheung, "BILL-E: Robotic Platform for Locomotion and Manipulation of Lightweight Space Structures," in AIAA Sci-Tech, 2017.

[13] M. Mikulas and J. T. Dorsey, "An integrated in-space construction facility for the $21 \mathrm{st}$ century," NASA Tech. Memo. 101515, 1988.

[14] S. Reza et al., "Aerocapture Inflatable Decelerator ( AID ) for Planetary Entry," Syst. Technol., no. May, pp. 1-18, 2007.

[15] U. Trabrandt, H. Koehler, and M. Schmid, "Deployable CMC Hot Structure Decelerator for Aerobrake," in 17th AIAA Aerodynamic Decelerator Systems Technology Conference, 2003.

[16] J. T. Dorsey and M. M. Mikulas, "Preliminary Design of a Large Tetrahedral Truss/Hexagonal Panel Aerobrake Structural System," in 31st Structures, Structural Dynamics and Materials Conference, 1990.

[17] S. J. Hughes et al., "Inflatable re-entry vehicle experiment (IRVE) design overview," 18th Aerodyn. Decelerator Syst. Technol. Conf., p. 2005, 2005.

[18] P. R. Savino et al., "European Sounding Rocket Experiment on Hypersonic Deployable Re-Entry Demonstrator," in Proceedings of 8th European Symposium on Aerothermodynamics for Space Vehicles, 2015.

[19] W. H. Greene, "Effects of Random Member Length Errors on the Accuracy and Internal Loads of Truss Antennas," J. Spacecr., vol. 22, no. 5, 1984.

[20] M. M. Mikulas, T. J. Collins, and J. Hedgepth, "Preliminary Design Approach for Large High Precision Segmented Reflectors," 1990.

[21] M. M. Mikulas, T. J. Collins, and J. Hedgepth, "Preliminary Design Considerations for 10-40 Meter-Diameter Precision Truss Reflectors," J. Spacecr., vol. 28, no. 4, 1991. 
[22] M. S. Lake, “Launching a 25-meter space telescope. Are astronauts a key to the next technically logical step after NGST?," in IEEE Aerospace Conference Proceedings, 2001.

[23] K. C. Wu and M. S. Lake, "Natural Frequency of Uniform and Optimized Tetrahedral Truss Platforms," 1994.

[24] M. S. Lake, L. Peterson, and M. Levine, "Rationale for Defining Structural Requirements for Large Space Telescopes," J. Spacecr. Rockets, vol. 39, no. 5, 2002.

[25] J. Nella et al., "James Webb Space Telescope (JWST) Observatory architecture and performance," Proc. SPIE, vol. 5487. pp. 576-587, 2004.

[26] M. W. Thomson, "The AstroMesh deployable reflector," IEEE Antennas Propag. Soc. Int. Symp. 1999 Dig. Held conjunction with Usn. Natl. Radio Sci. Meet. (Cat. No.99CH37010), vol. 3, pp. 15161519, 1999.

[27] A. Meguro, K. Shintate, M. Usui, and A. Tsujihata, "In-orbit deployment characteristics of large deployable antenna reflector onboard Engineering Test Satellite VIII," Acta Astronaut., vol. 65, no. 910, pp. 1306-1316, 2009.

[28] H. Bush and et al, "Design and Fabrication of an Erectable Truss for Precision Segmented Reflector Application," J. Spacecr., vol. 28, no. 2, 1991.

[29] J. M. Hedgepeth, "Influence of Fabrication Tolerances on the Surface Accuracy of Large Antenna Structures," AIAA J., vol. 20, no. 5, pp. 680686, 1982.

[30] W. H. Greene, "Effects of random member length errors on the accuracy and internal loads of truss antennas," vol. 22, no. 5, pp. 554-559, 1983.

[31] S. Calisch, "Physical Finite Elements," Massachusetts Institute of Technology, 2014.

[32] J. Hiller and H. Lipson, "Dynamic Simulation of Soft Multimaterial 3D-Printed Objects," Soft Robot., vol. 1, no. 1, pp. 88-101, 2014.

[33] M. F. Ashby, "The properties of foams and lattices.," Philos. Trans. A. Math. Phys. Eng. Sci., vol. 364, no. 1838, pp. 15-30, 2006.

[34] L. Puig, A. Barton, and N. Rando, "A review on large deployable structures for astrophysics missions," Acta Astronautica, vol. 67, no. 1-2. pp. 12-26, 2010.

\section{BIOGRAPHY}

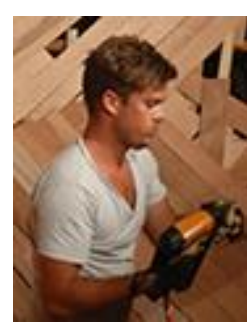

Benjamin Jenett is a graduate student researcher at MIT's Center for Bits and Atoms, where he is pursuing his $\mathrm{PhD}$ on automated assembly for large aerospace structures. $\mathrm{He}$ is a NASA Space Technology Research Fellow.

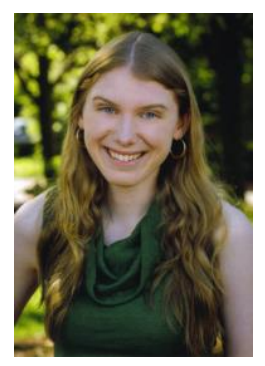

Christine Gregg is a graduate student researcher in the Advanced Manufacturing for Energy group in the Department of Mechanical Engineering at UC Berkeley, where she is pursuing her $\mathrm{PhD}$ on digital lattice structures and their fracture mechanics. She is a NASA Space Technology Research Fellow.

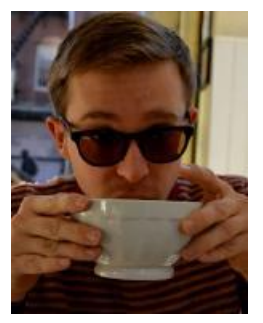

Daniel Cellucci is a Ph.D candidate studying Mechanical Engineering at Cornell University. He conducts research on digital material structures and relative robots. He is a NASA Space Technology Research Fellow.

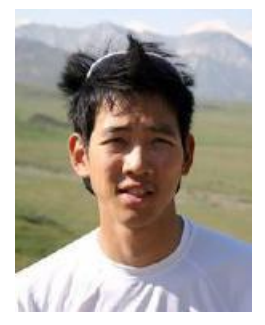

Kenneth Cheung received his Ph.D. from the Center for Bits and Atoms at the Massachusetts Institute of Technology. $\mathrm{He}$ helps to run the ARC Coded Structures Laboratory (CSL), which conducts research on the application of building block based materials and algorithms to aeronautical and space systems. As a member of the Ames CCT staff, he serves as the technical lead on advanced materials and manufacturing. 